\title{
Design of Rotary Tillage Work Tools as Per Agro- Ecological Requirements
}

\author{
Yu. F. Kazakov, V. I. Medvedev, P. V. Mishin \\ Department of "Transport and technological machines and \\ complexes" \\ Chuvash state agricultural Academy \\ Cheboksary, Russian Federation \\ ura.kazakov@mail.ru \\ N. V. Obolensky \\ Engineering faculty \\ Nizhny Novgorod state University of engineering and \\ Economics \\ Nizhny Novgorod region, Russia \\ obolenskinv@mail.ru
}

\author{
P. V. Zaitsev, V. V. Belov, S. A. Ovchukova \\ The Department of "Mechanization, electrification and \\ automation of agricultural production" \\ Chuvash state agricultural Academy \\ Cheboksary, Russian Federation \\ ovchukova1941@mail.ru
}

\author{
G. S. Yunusov \\ Department of Mechanization of Production and Processing \\ of Agricultural Products \\ Mari State University \\ Yoshkar-Ola, Russia \\ prk@marsu.ru
}

axial load [1,6-8], the need for high-kinematic operation mode [3], limited resource of work in areas littered with stones [5,9];

The use of narrow-cut vane rotary work tools for the formation of furrows and grass seeds sowing in the sod layer promotes protection from water erosion when slope tillage. Methods to form an irregular curve blade working surface are proposed. It is a sector of the closed helical surface of constant or variable steps. A set of blades with different surface geometries is obtained by changing the inclination angle of the reproducing line, and the value and direction of the velocity of its motion along the axis of the cylindroid by setting various radial and axial steps of helicoidal lines. Tooling of rotary tillage work tools with the bladed working elements in the form of helicoidal surfaces with variable pitch, forming the alternating stress-strain state in the disposed cut, contributes to the reduction of energy consumption, enhancing their functionality.

Keywords - sod layer; trench plough; blade; design

\section{INTRODUCTION}

When improved forage lands on the slopes, most relevant technologies, complying with agri-environmental requirements, are those including operations of grass seeds underseeding and mineral fertilization in the sod layer [1,2]. Quality formation of grooves in the sod layer is provided by active disks, operating at high values of the ratio of the circumferential and forward velocity [1,3-7], expressed by the kinematic factor.

\section{II.THE LITERARY DATA ANALYSIS, GOAL SETTING AND OBJECTIVES OF THE STUDY}

The following is applied for the formation of grooves in the sod layer:

- drive round oscillating disks [2,3], equipped with carbides [2,5], providing landing. Their disadvantages: uneven bottom of open grooves $[1,4]$, the fluctuating
- flat toothed elliptical swing discs, which advantage is constant circumferential cutting speed, smooth plow sole along its width, but requiring a high kinematic operation mode $[1,10]$.

- trench plough in the form of a hub which has quadrant elliptical flat blades evenly located around the circumference. The major semi-axis of each blade is inclined from the vertical for an angle, and minor semiaxis are in a longitudinal vertical plane of motion (Fig. 1) $[1,10]$. The marginal blade is made along the edge of the blade, and the stand blade lines up with its minor axis.

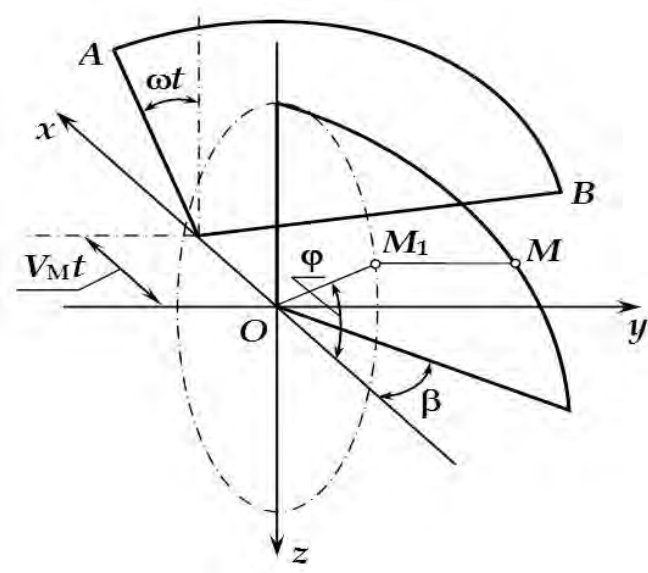

Fig. 1. The blade in the initial and current times 
The module of the velocity vector is equal to:

$$
V_{a}=\sqrt{\rho^{2} \omega^{2}+2 \rho \omega \sin (\varphi+\alpha)+V_{M}}
$$

The absolute velocity $\mathrm{V}_{\mathrm{a}}$ depends on the angle of rotation of the work tool $(\alpha=\omega t)$, the ratio of the semi-axis of elliptical blade $\beta$, the distance from the axis of rotation $\rho$ along the minor semi-axis, kinematic operation mode $\lambda=\omega \rho^{2} / V_{M}$, parameter $\varphi$ - the angular distance of the major semi-axis to the current point of the blade.

Fig. $2 \mathrm{a}$ and $2 \mathrm{~b}$ show the dependences of va on various factors.

Figure 2a shows that the effect of parameter $\varphi$ on the value of the velocity is most significant at $\varphi=0^{0}$. The smallest value of the absolute velocity (Fig. $2 a$ ) happens when interaction between the soil and the blade area are adjacent to the major semi-axis, that is, when $\varphi=90^{\circ}$. Velocity value at the moment is due to the ratio of the circumferential and forward velocities of the work tool (figure $2 a$ represents it as the minimum velocity), and does not depend on parameter $\beta$.

Increased rotation frequency of the work tool to improve the quality of soil pulverization is accompanied by an increase in absolute velocity, and hence energy consumption. In addition, the attempt to increase productivity by expanding the work tool coverage will require increasing $\beta$, which also leads to increased speed and, consequently, energy consumption.

The analysis showed that differential blade surface parameters change in its different areas, implementation of alternate stress-strain in the subtract cut are required, that is, a non plane working surface of the blade is needed $[12,14,15]$. The alternation of concave and convex areas of the blade needs to be provided along its parallel and meridian [12]

This achieves a consistent effect on the cut by blade areas with an opposite signs curvature as along the cut (along the main blade - parallel) and crosswise of the cut (along the meridian). To perform various agricultural operations, it is necessary to provide different intensity of curvature change with the localization of inflection point with different combination of design parameters given the relative landing of the work tool $[1,12]$.

It is the purpose of this paper to develop a designing method of a non-plane working surface of the blade, providing reduction of energy consumption and expanding of the agrotechnical opportunities of the work tool.

\section{III.A DESIGNING METHOD OF THE BLADE SURFACE}

The concave-convex blade surface is formed in two ways: by the motion of a point on three axes and motion of the generatrix of different types along various guiding lines $[12,15]$. In the first method, the result depends on the ratio of the velocities value and motion direction relative to the axes. In the second blade surface forming method, helicoidal lines are used as the guiding lines. Type of generatix, velocity and its direction affect the change intensity of concavity and convexity of the blade as a whole and its individual functional areas. If a rectilinear segment is used as a generatrix, the line segment, and the uniform helix serves as a guiding line, then the resulting surface will be flat.

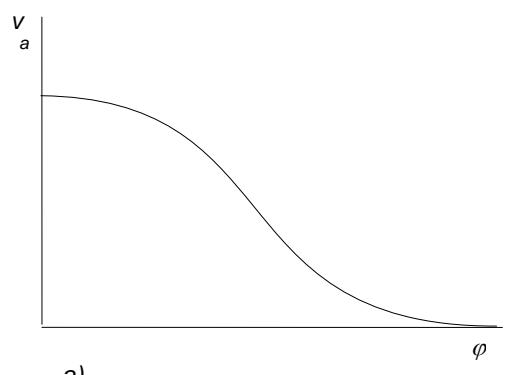

a)

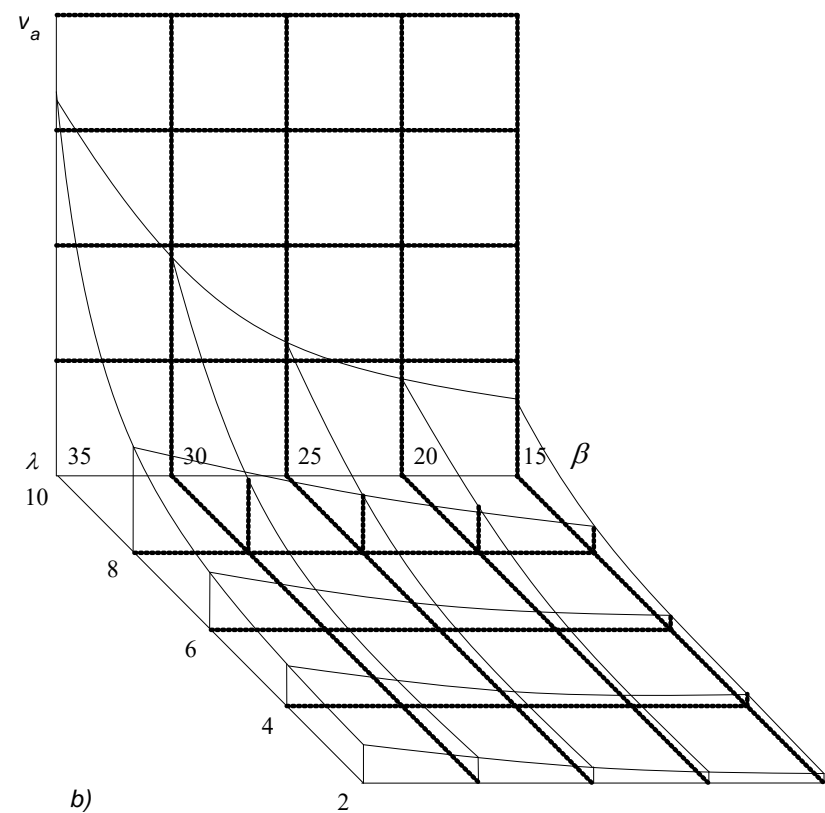

Fig. 2. The change of absolute velocity $V_{a}$ of the soil particle: a depending on the blade area acting on the particle of the soil (parameter $\varphi) ; b$ - depending on the kinematic coefficient $\lambda$ and the ratio of blade elliptical semi-axis $\beta$, degr.

There are various ways of setting parameters of the guiding lines: interpolation, approximation, geometric design, parametric and analytical. Approximation can be conducted using Bezier method or spline functions. Geometric design can be implemented through the method "of progressica". Parametrically defined guiding lines (curves of Ferguson, Alymov), often used in the design of ship forms, provide a significant extension of functional capabilities of the work tools. The assets of a developer of non-plane blades also consist of analytical curves of the second order and exponential functions, successfully applied in the design of the aircraft forms and the working surface of internal combustion engines gas distribution mechanism cams.

Reproducing line motion velocity change along the screw axis, and the inclination angle of reproducing the line to this axis (the latter is achieved by adjusting the corresponding helix parameters of helix), we set the patterns of signs change and the curvature change rate as per the parallel and meridian of the 
blade. Formed surface is a sector of the closed helical surface of constant or variable steps. When using generatrix coaxial to the cylinder helical lines (circular, elliptical, etc.) as the third guiding line, we will receive a surface as an open helical surface of variable or constant pitch.

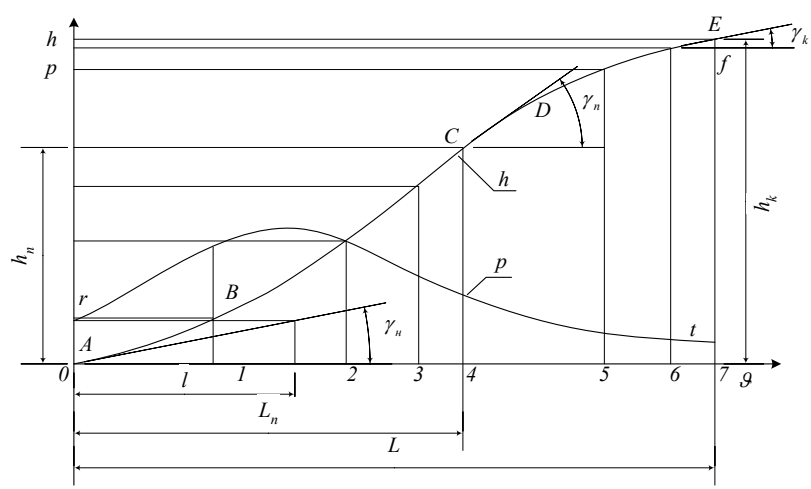

Fig. 3. The change in the lift value $h$ of the generating line and of the parameter $p$ of the helical surface depending on the rotation angle $\vartheta$ of the generating line: $\mathrm{L}$ is the length of the marginal blade; $h \mathrm{~h}$ is the deviation of the major semi-axis (furrow width); $\mathrm{Ln}$ is the length of the blade, corresponding to the localization of the inflection point on the blade surface; $\mathrm{hn}$ is the blade surface deviation from the minor semi-axis rotation plane corresponding to the inflection point

A set of blades with different surface geometry is obtained by changing the inclination angle of the reproducing line, velocity of its motion along the axis of the cylindroid by setting various radial and axial steps of helicoidal lines. The lift $h$ of the generating line along the axis of the helical lines is set depending on the angle of its rotation $\vartheta$ (Fig. 3).

To determine the parameter $\mathrm{p}$ of the helical surface at any its point, we trace tangency to the corresponding profile curve point Of, corresponding to the concave-convex surface. The tangent of a tangency angle to the $\mathrm{x}$-axis $\quad p=\lim \frac{\Delta h_{\vartheta}}{\Delta \vartheta}$ will be equal to the parameter of the surface points of reproducing the straight line at its corresponding position. For a number of points of the curve, let us determine the value $p$, build curve rt in the adopted scale unit. It describes the helix parameter $p$ change of the surface depending on the rotation angle $\vartheta$.

The values $h$ and $p$, characterizing the profile line Of, are features forming, and shall be justified on the basis of agroecological requirements for a particular operation when tillage of the slopes.

The blade surface, allowing formation of the alternate stress in cuts, is also obtained by a combination of two or three singleband blades. The angles of reproducing line (rectilinear segment) and axial steps of the guiding line (helix parameters p) will be different for each pair of helicoidal lines (Fig.4)

\section{A. The design results of the non-plane blade and their analysis}

Distinctive combinations of helix parameters - ratio of velocities of forward and rotational movements of the generatrix of the helical surface of the inner $p_{1}$ and outer $p_{2}$ of helicoidal lines, are shown in fig. 4.

Mainly the removal of soil from the furrow and its tailings at a considerable distance is reached, if the ratio of helix parameters $\mathrm{P} 1>\mathrm{P} 2$, shown in Fig. $4 \mathrm{a}$, is implemented. When the ratio $\mathrm{p} 1<\mathrm{p} 2$ (Fig. 4 b), the blade will move the layer (cut) with the rotation round the layer axis parallel to the vector of forward velocity of the tool and put it next to the open furrow. If the helix parameter of the internal helicoidal line is slightly higher than the similar value of the outer helicoidal line, it is possible to achieve moderate removal of soil at a constant rotation frequency of the work tool. These ratios are recommended for the work tools, designed for cutting furrows in the sod layer for rejuvenation of the plant formation, and enhance the decomposition of organic substances for subsequent sowing grasses seeds and mineral fertilizers embedding. Such ratio implementation is also preferable when the design of the work tool for the formation of the stitches before potato planting and inter-row tillage. A ration change $\mathrm{P} 1>\mathrm{P} 2$ for $\mathrm{P} 1<\mathrm{P} 2$ and vice versa along the blade and along the slice of furrow (Fig. 4 b, 4 d) is intended to form the alternating stress - strain state in disposed cut, thereby reducing energy consumption for tillage [12].
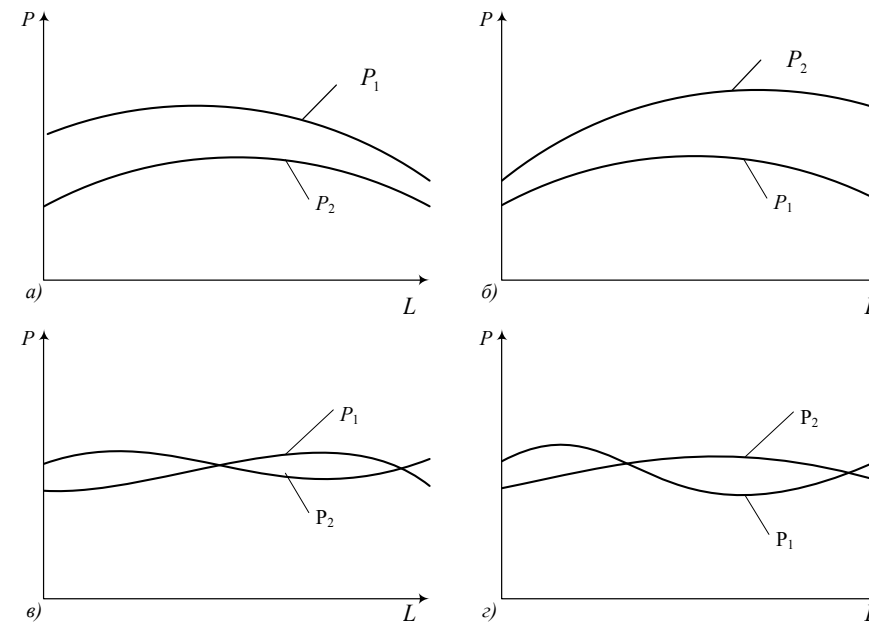

Fig. 4. Changing of the helical lines parameters $\mathrm{p}_{1}$ and $\mathrm{p}_{2}$ along the length $L$ of the blade.

Fig. 5 shows graphs of motion velocity change of the generating line along the axis of the helix (along a generatrix of the cylinder - in the case of an open helicoidal surface).
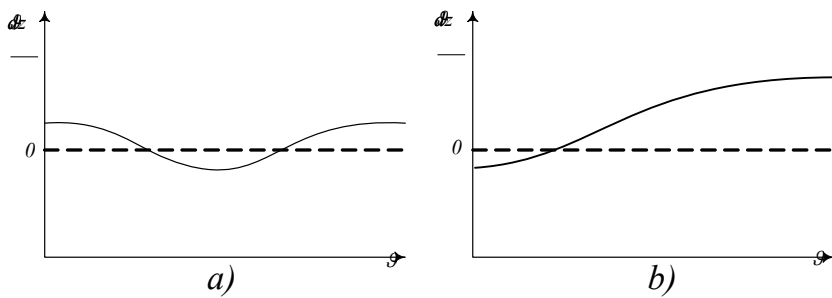

Fig. 5. Motion velocity change of the generatrix $d z / d \vartheta$ along the axis of the screw depending on the generating line rotation angle $\vartheta: a$ - along the axis of the helix lines; $b$ - along a generatrix of the cylinder - - - - for flat blade; ----- for blade of variable curvature. 
Impact of different areas on the soil (Fig. 5 a) provides laying of soil to a wall of cut stitch with low speed, avoiding choking of hilling plants (Fig. 5 b).

For a visual representation of the blade surface at different distances from the axis (along the Meridian) and the plane, sweeping by the minor semi-axis, we use skeletons - blade helical sections (Fig. 6). They are obtained by a cross section of the blade coaxial to the screw axis by the cylinders of appropriate radii. The blade section base or the so-called chord of the profile is the position of the generatrix. In the crosssection, longitudinal profile of the line along the parallel blades (stroke thickness) will be obtained with the coaxial cylinders, and in the cross-section along the generating line-profile lines along the Meridian - from the daylight soil surface to the furrow sole.

Cross-sections of blades by planes parallel to the axis of the screw at a particular angular distance from the minor semi-axis rotation plane are more typical for the tillage work tool blades. As a result of proper coordination of the above-mentioned sections, we receive a combination of longitudinal and transverse profile lines for specific sectors of the blade, for example, "polydyne" or Alymov's curves.

Fig. 6 shows cross-sections of the flat surface of the blade, differing in the angle of inclination of the generatrix to the axis of the screw. Changes of the non-plane blade surface shape from the moment of blade entry into the soil up to its out are shown in figures $6 \mathrm{~b}$ and $6 \mathrm{c}$. The change pattern of the sagitta of segments $\Delta 1 \ldots \Delta 5$ (as moving away from the minor blade semi-axis) is caused by agro-requirements for the soil tillage operation. The blade area, limited by the angle $\beta \mathrm{H}$, characterized in figures $6 \mathrm{~b}$ and $6 \mathrm{c}$ with different curvature signs, gives an idea on the change in the stress-strain state in the cutting chips, on the nature prevailing stress in it: compression stresses (Fig. 6 b) and tension stresses (Fig. 6 c). As to further interaction of the subsequent blade areas with cuts, due to a change of blade curvature sign, there will be appropriate changes and prevailing stresses in the cut. The nature of the curvature change in the final blades sections, characterized by the sagitta of segments $\Delta 4$ and $\Delta 5$, plays a decisive role in shaping the direction and range of soil particles.

For example, when forming a furrow in the sod layer for grass seed sowing it is desirable to achieve the patterns shown in Fig. 6. The necessary change pattern of sagitta of a segment and shape of the blade working surface with the purpose of cutting niches for subsequent self-falling of their walls and ensure the decompression of sod layer in the improved area of the grassland, is shown in Fig. 6 b. Fig. 6 shows the sequence of sagitta of segments changes for the formation of waterretaining elevation on the slopes by cutting strips and formation of soil embankments.
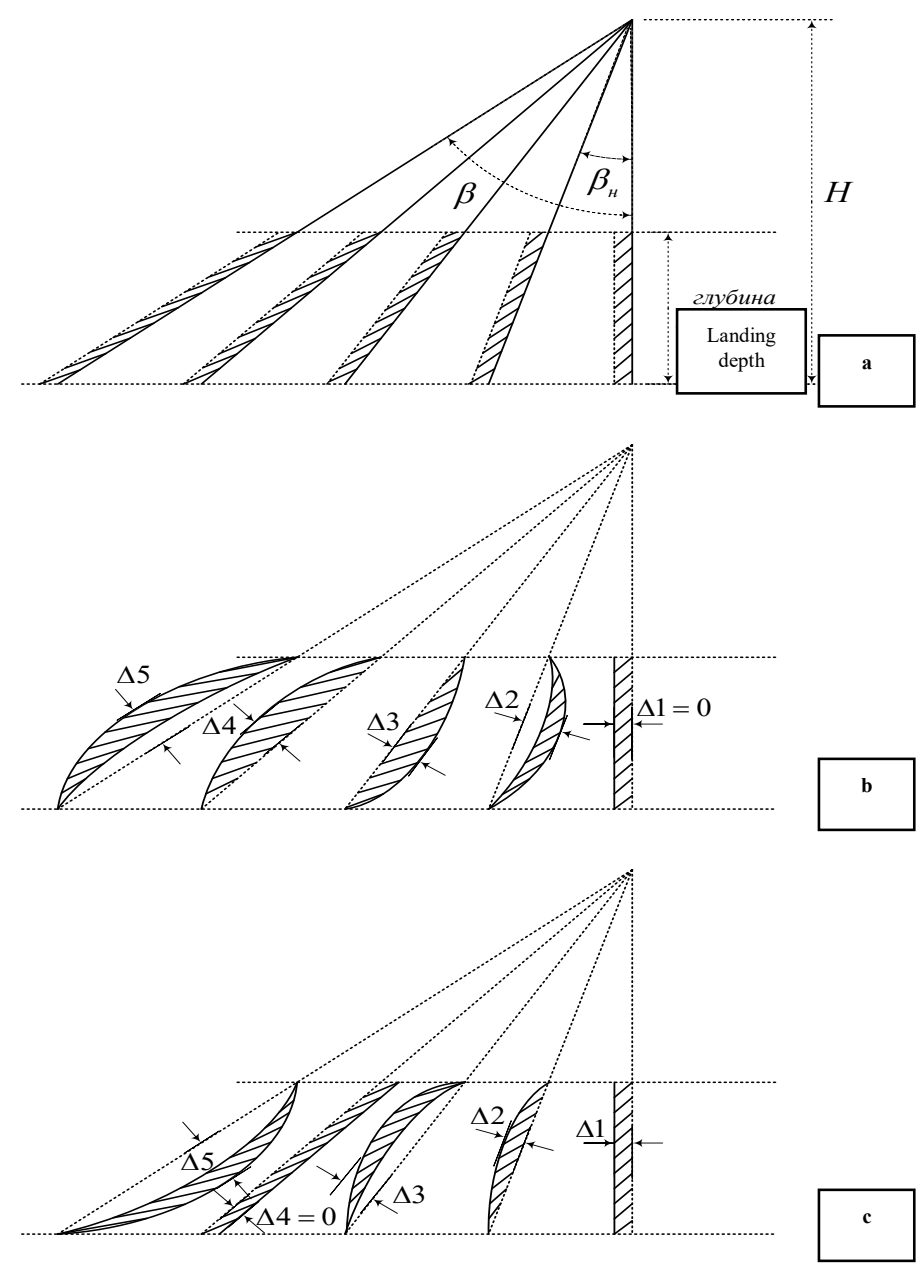

Fig. 6. The blade surface change scheme depending on the purpose of rotating work tool during the lift of the helical line to the height $\mathrm{H}$, corresponding to the duration of the interaction of the blades with the soil.

\section{CONCLUSION}

The methodology for the design of the working surface of trench plough non-plane blade, allowing implementing the alternating stress-strain state in the cut soil chip, is proposed.

Tooling of rotary tillage work tools with the bladed working elements in the form of helicoidal surfaces with variable pitch contributes to reducing energy consumption, enhancing their functionality, ensures the implementation of the soil-protective energy - and material-saving technologies for improvement of grassland on the slopes.

\section{References}

[1] Yu. F. Kazakov, "Technology and means of mechanization for the productivity of the grassland", Agricultural mechanics at the turn of the century. Scientific. Tr., Tauric. state agrotechnical Academy, Melitopol, vol. 22, 2001, No. 1, pp. 132-137.

[2] P. Guo, M.A. Choudhary, "Preliminary studies of a modified slot opener for direct drilling seeds", New Zealand J. of Experimental Agriculture, Vol. 13, 1985, No. 1, pp. 85-95. 
[3] L.G. Wells, E.M. Smith, D.E. Hammett, "Analyses and Testing of Powered Tillage Blades", Trans. of the ASAE, Vol. 23, 1980, No. 6, pp. 1383-1387.

[4] L.G. Wells, E.M. Smith, D.E. Hammett, H.J. Tompson, "Ground Driven Powered Tillage", Trans. of the ASAE, Vol. 23, 1980, No. 6, pp. 1379 1382.

[5] J. Howe, "Sod Seeders on Trial", Power Farming, vol. 11, 1981, pp. 8-11.

[6] J. Howe "Sod Seeders on Trial: the Results", Power Farming, vol. 9, 1982, pp. 28-29.

[7] S. V. Yanushko, "Increasing the productivity of hayfields and pastures sowing seeds in the sod of perennial legumes", Gorki: Intensive technology of cultivation of forage crops in the conditions of the BSSR, 1988, pp. $6-11$.

[8] T.C. Thakur, R.J. Godwin, "The mechanic of soil cutting by a rotation wire", J. Terramechanics, vol. 27, 1990, No. 4, pp. 291-305.

[9] T.C. Thakur, R.J. Godwin, "The present state of force prediction models for rotary powered tillage tools", J. Terramechanics, vol. 26, No. 2, 1989, pp. 121-138.

[10] S. Yonekawa, O. Kitani, T. Okamoto, N. Sakai, "Studies on the Measuring System of a Soil - Tillage Tool Interaction (Part 3) -
Measurement of Soil Stresses and Displacements in Tillage with a Rotational Motion Blade and Double Blades", J. of the Japanese Society of Agricultural Machinery, vol. 53, 1991 , No. 2, pp. 33-39.

[11] V. A Sysuev., A. D. Kormschikov, A. M. Pjatin, A. S. Ovsyannikov, "Technology and technical means for band seeding grasses in sod" Kirov: NIISKH Severo-Vostoka, 2000, p. 60.

[12] Yu. F. Kazakov, "Justification of parameters of the spiral work items soil cutters", Bulletin of the Chuvash state agricultural Academy, vol. 2, 2017, pp. 68-74.

[13] M. N. Chatkin, "Kinematics and dynamics of rotational soil-cultivating working bodies of screw elements", Saransk: Mordov state University, 2008, p. 314.

[14] V. I. Vetokhin, "Justification of the form and parameters of loosening working bodies in order to reduce energy consumption for soil treatment", dissertation for Cand. of Techn. Sciences, Moscow: VISKHOM, 1982, p. 232 .

[15] A.I. Leschankin, "Design of rotary tillage working bodies", Saransk: Mordov. state University, 1989, p. 92 